\title{
Narrow-wall slotted waveguide array antenna with low cross-polarization filter
}

\author{
Guoan Xiong ${ }^{1, a}$, Jin $\operatorname{Pan}^{1, b}$ and Boyuan $\mathrm{Ma}^{2, \mathrm{c}}$ \\ ${ }^{1}$ School of Electronic Engineering, University of Electronic Science and Technology of China, \\ Chengdu 611731, China; \\ ${ }^{2}$ Yingcai Honor College, University of Electronic Science and Technology of China, Chengdu \\ 611731, China; \\ agustave_xiong@foxmail.com, bpanjin@uestc.edu.cn, c331953406@qq.com
}

Keywords: slotted waveguide antenna, array, cross-polarization, filter.

\begin{abstract}
A narrow-wall slotted waveguide array antenna with low cross-polarization filter is presented. The horizontal polarization (HP) is the co-polarization for narrow-wall slotted waveguide array antenna, while the vertical polarization (VP) is the cross-polarization. High level cross-polarization reduces effect and is undesirable. Some formulas were presented to give a concise explanation how this structure reduces cross-polarization. On this basis, a 32-element low cross-polarization narrow-wall slotted waveguide array antenna in $9.305 \mathrm{GHz}$ was designed and simulated. The antenna has the characteristics SLL=-20dB and cross-polarization lower than $-40 \mathrm{~dB}$.
\end{abstract}

\section{Introduction}

The slotted waveguide array antenna is used in radar technology and communications because of high gain performance, low level secondary beams, manufacture cost and low loss. Slots are fabricated in narrow walls or wide walls. For narrow-wall slotted waveguide array antenna, the slant slots with different angles and depths must interrupt the surface currents to radiate $\mathrm{TE}_{10}$ mode wave. The slant slots mainly radiate horizontal polarization wave, while vertical polarization wave is also radiated from them. Cross-polarization in such antenna is not neglectable, which cause additional lobs in azimuth pattern.

For the purpose of decreasing undesired cross-polarization in narrow-wall slotted waveguide array antenna, some methods have been presented. Using polarization filter is the simplest way to reduce cross-polarization [1]. A kind of polarization filter is consisted of orthogonal walls between slots. By choosing distance between two adjacent orthogonal walls, vertical polarization can be decreased. Another simple polarization filter was also developed. It is consisted of a layer that many metal strips are printed [2]. By choosing width of strips, distance between metal orthogonal strips and thickness of layer, this filter has minimal influence on horizontal polarization while significant influence on crosspolarization. Many new structures were developed to decrease cross-polarization. A pair of shaped irises that flanks the slot in the waveguide is designed to reduce cross-polarization level [3]. The cross-polarization level is less than $-40 \mathrm{~dB}$ in an array with 16 such elements. By inserting slant ridge under the slots, slots can be erect which only radiate horizontal polarization signal [4].

Based on recent reports, it seems that most of the works reported on low cross-polarization narrow-wall slotted waveguide array antenna have focused on methods to design it. But the physical principle and calculation method are not reported systematically. In this paper, the narrow wall slotted waveguide array antenna with low cross-polarization rectangular waveguide filter is designed and simulated by HFSS. The designed array antenna has characteristics cross-polarization lower than $-40 \mathrm{~dB}$ and SLL=-20dB. 


\section{Antenna Design}

\subsection{Polarization Filter Design.}
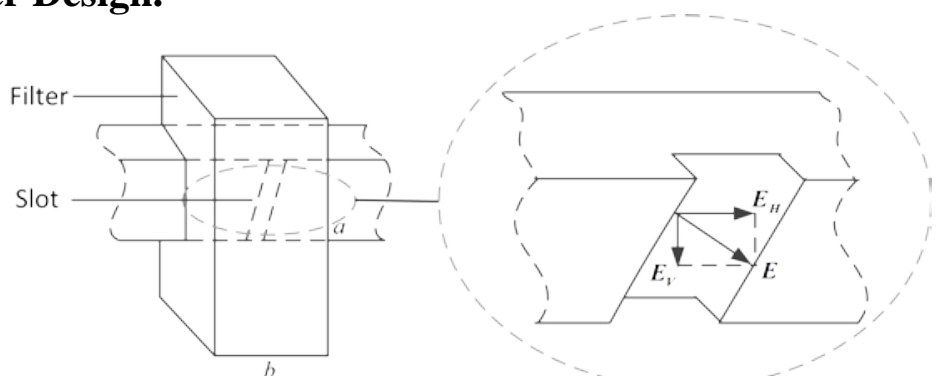

Fig. 1 Narrow-wall slotted waveguide array antenna with rectangular waveguide polarization filter and its E field distribution

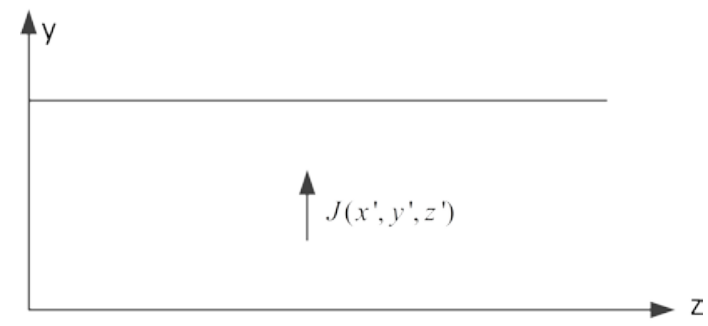

Fig. 2 Time-harmonic current in semi-unbounded rectangular waveguide system

The rectangular waveguide polarization filter is shown in Fig.1. By means of Babinet's principle [5], many of the problems of slot antennas including slotted waveguide antenna can be reduced to situations solving complementary linear antennas for which solutions have already been obtained. For narrow wall slotted waveguide array antenna, E field is shown in Fig. 1. In Fig. 1, E field can be decomposed into $\boldsymbol{E}_{\boldsymbol{H}}$ and $\boldsymbol{E}_{\boldsymbol{V}}$. By the means of Babinet's principle, $\boldsymbol{E}_{\boldsymbol{H}}$ can be excited by $\boldsymbol{I}_{\boldsymbol{H}}$ in horizontal electric dipole antenna, and $\boldsymbol{E}_{\boldsymbol{V}}$ can be excited by $\boldsymbol{I}_{\boldsymbol{V}}$ in vertical electric dipole antenna.

In a waveguide system, when the operating frequency is less than the cutoff frequency of $\mathrm{TE}_{01}$ wave $\left(f<f_{c}\right), T E_{01}$ wave mustn't propagated. There is the same $E$ field distribution between $\mathrm{TE}_{01}$ wave in a rectangular waveguide system and vertical electric dipole antenna. For the purpose of reducing $\boldsymbol{E}_{\boldsymbol{V}}$ (cross-polarization), the condition as bellow must be fulfilled to ensure the falloff of $\mathrm{TE}_{01}$ mood,

$$
b<\lambda / 2
$$

where: b-length of narrow wall of waveguide filter; $\lambda$-length of electromagnetic in a free space

To ensure the propagation of $\mathrm{TE}_{10} \operatorname{mood}$ (co-polarization), the condition as bellow must be fulfilled

$$
a>\lambda / 2
$$

where: a-length of broad wall of waveguide filter; $\lambda$-length of electromagnetic in a free space

In order to ensure the cross-polarization field in this kind of system, slots can be regarded as the excitation source for above semi-unbounded rectangular waveguide system in Fig. 2. E field excited by y direction current $\boldsymbol{J}\left(\boldsymbol{r}^{\prime}\right)$ in (x', $\left.\mathrm{y}^{\prime}, \mathrm{z}^{\prime}\right)$ can be presented by electric dyadic Green function of the first kind as follows

$$
\boldsymbol{E}(\boldsymbol{r})=-j \omega \mu \int_{V} \overline{\overline{G_{1}^{e}}}\left(\boldsymbol{r}, \boldsymbol{r}^{\prime}\right) \cdot \boldsymbol{J}\left(\boldsymbol{r}^{\prime}\right) d V^{\prime}
$$

By dyadic Green functions in electromagnetic theory, we obtain

$$
\nabla \times \nabla \times \overline{\overline{G_{1}^{e}}}\left(\boldsymbol{r}, \boldsymbol{r}^{\prime}\right)-k^{2} \overline{\overline{G_{1}^{e}}}\left(\boldsymbol{r}, \boldsymbol{r}^{\prime}\right)=-\boldsymbol{e}_{\boldsymbol{y}} \boldsymbol{e}_{\boldsymbol{y}} \delta\left(r-r^{\prime}\right)
$$

The direction of the vector magnetic potential excited by y direction current $\boldsymbol{J}\left(\boldsymbol{r}^{\prime}\right)$ in waveguide is along with y axis. Thus, this problem can be simplified using potential dyadic Green function $\overline{\bar{G}}\left(\boldsymbol{r}, \boldsymbol{r}^{\prime}\right)$ and potential scalar Green function by (5)-(7). The boundary conditions are shown in (8) 


$$
\begin{aligned}
& \overline{\overline{G_{1}^{e}}}\left(\boldsymbol{r}, \boldsymbol{r}^{\prime}\right)=\left(1+\frac{1}{k^{2}} \nabla \nabla\right) \overline{\bar{G}}\left(\boldsymbol{r}, \boldsymbol{r}^{\prime}\right) \\
& \overline{\bar{G}}\left(\boldsymbol{r}, \boldsymbol{r}^{\prime}\right)=\boldsymbol{e}_{y} \boldsymbol{e}_{y} G\left(\boldsymbol{r}, \boldsymbol{r}^{\prime}\right) \\
& \nabla \cdot \nabla G\left(\boldsymbol{r}, \boldsymbol{r}^{\prime}\right)+k^{2} G\left(\boldsymbol{r}, \boldsymbol{r}^{\prime}\right)=-\delta\left(\boldsymbol{x}-\boldsymbol{x}^{\prime}\right) \delta\left(\boldsymbol{y}-\boldsymbol{y}^{\prime}\right) \delta\left(\mathbf{z}-\boldsymbol{z}^{\prime}\right) \\
& \left\{\begin{array}{l}
\left.G\left(\boldsymbol{r}, \boldsymbol{r}^{\prime}\right)\right|_{x=0, a ; z=-L}=0 \\
\left.\frac{\partial G\left(\boldsymbol{r}, \boldsymbol{r}^{\prime}\right)}{\partial y}\right|_{y=0, b}=0
\end{array}\right.
\end{aligned}
$$

By method of ohm-RayLeigh, potential scalar Green function $G\left(\boldsymbol{r}, \boldsymbol{r}^{\prime}\right)$ can be presented as (9), so using (5)-(6), electric dyadic Green function $\overline{\overline{G_{1}^{e}}}\left(\boldsymbol{r}, \boldsymbol{r}^{\prime}\right)$ is shown in (10). The co-polarization E field excited by slots in rectangular waveguide system can be calculated from (3)

$$
\begin{aligned}
& G\left(\boldsymbol{r}, \boldsymbol{r}^{\prime}\right)=\frac{2}{a b} \sum_{m=1}^{\infty} \sum_{n=0}^{\infty} \frac{2-\delta_{n 0}}{\gamma_{m n}} \sin \frac{n \pi x}{a} \sin \frac{n \pi x^{\prime}}{a} \cos \frac{m \pi y}{b} \cos \frac{m \pi y^{\prime}}{b} \cdot \begin{cases}e^{-\gamma_{m z^{\prime}}} \operatorname{sh} \gamma_{m n} z & z \leq z^{\prime} \\
e^{-\gamma_{m n} z} \operatorname{sh} \gamma_{m n} z^{\prime} & z \geq z^{\prime}\end{cases} \\
& \overline{\overline{G_{1}^{e}}}\left(\boldsymbol{r}, \boldsymbol{r}^{\prime}\right)=\boldsymbol{e}_{x} \boldsymbol{e}_{y} \frac{1}{k^{2}} \frac{\partial^{2} G\left(\boldsymbol{r}, \boldsymbol{r}^{\prime}\right)}{\partial x \partial y}+\boldsymbol{e}_{y} \boldsymbol{e}_{y}\left[G\left(\boldsymbol{r}, \boldsymbol{r}^{\prime}\right)+\frac{1}{k^{2}} \frac{\partial^{2} G\left(\boldsymbol{r}, \boldsymbol{r}^{\prime}\right)}{\partial y^{2}}\right]+\boldsymbol{e}_{z} \boldsymbol{e}_{y} \frac{\partial^{2} G\left(\boldsymbol{r}, \boldsymbol{r}^{\prime}\right)}{\partial z \partial y} \\
& \text { where: } \gamma_{m n}^{2}=\left(\frac{m \pi}{a}\right)^{2}+\left(\frac{n \pi}{b}\right)^{2}-k^{2} ; \delta_{n 0}= \begin{cases}1 & n=0 \\
0 & \text { else }\end{cases}
\end{aligned}
$$

\subsection{Array antenna Design.}

Fig. 3 shows the structure of 32-element slotted waveguide array antenna with rectangular waveguide cross-polarization filter. $B J 100$ waveguide $(a=22.86 \mathrm{~mm}, b=10.16 \mathrm{~mm}, t=1.27 \mathrm{~mm})$ is chosen to be slotted. The filter is made up of FR4 layer with copper of $0.018 \mu \mathrm{m}$ on it. Table 1 shows the structure parameters. By Taylor synthesis method, the amplitude of each slot in 32 elements of $\mathrm{SLL}=20 \mathrm{~dB}$ can be get. The equivalent normalized conductivity distribution is also calculated by [6] as Fig. 4(a). The equivalent normalized conductivity distribution is the maximum at an angle among different depths. Using (11), the relationships among angle, equivalent normalized conductivity g and slot depth can be simulated by HFSS. The correspondence among angle, equivalent normalized conductivity g and slot depth can be shown in Fig. 4(b). Using Fig. 4(a) and 4(b), the angle and depth of each slot can be sure.

$$
g=e^{-2 \alpha d}\left(\frac{S_{12}^{2}}{1-S_{11}^{2}}\right)^{-\frac{1}{N}}-1
$$

where: $\alpha$-waveguide loss per unit length; $\mathrm{N}$-number of slots

Table 1 Structure Parameters

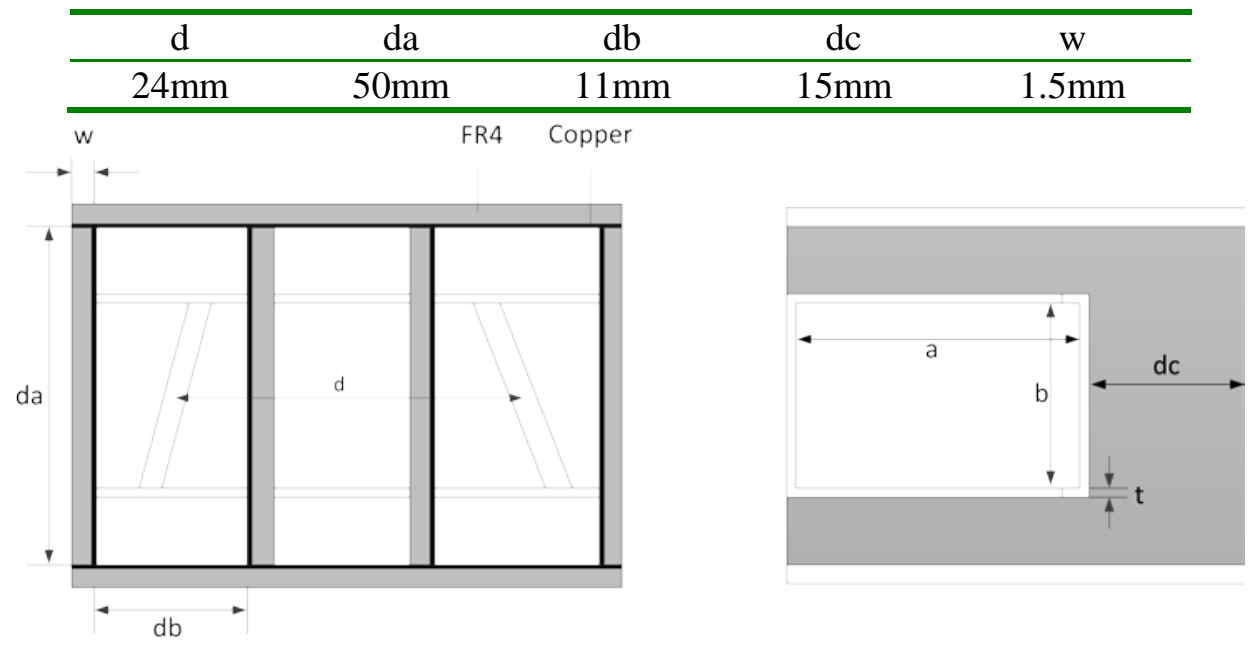

Fig. 3 Slotted waveguide array antenna with rectangular waveguide cross-polarization filter 
Fig. 5 shows the simulated radiation pattern in horizontal plane in 32-element slotted waveguide antenna with cross-polarization filter in $9.305 \mathrm{GHz}$. The simulated maximum side lobe level is - $20 \mathrm{~dB}$ with cross-polarization level of $-40 \mathrm{~dB}$ below the main lobe in the band.

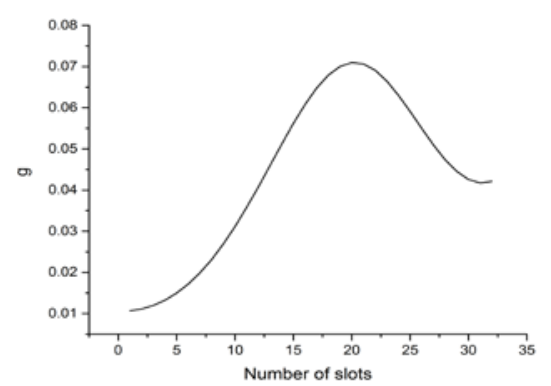

(a)

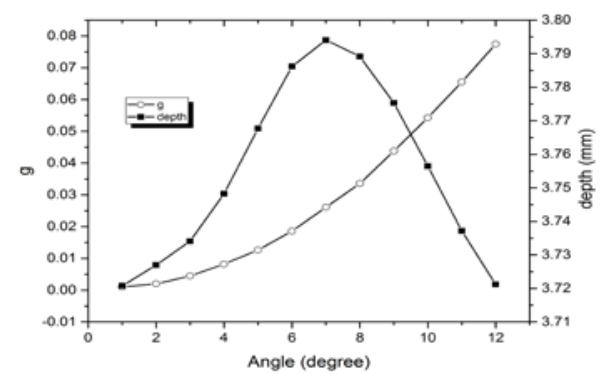

(b)

Fig. 4 (a) Equivalent normalized conductivity distribution (b) Correspondence among angle, equivalent normalized conductivity g and slot depth

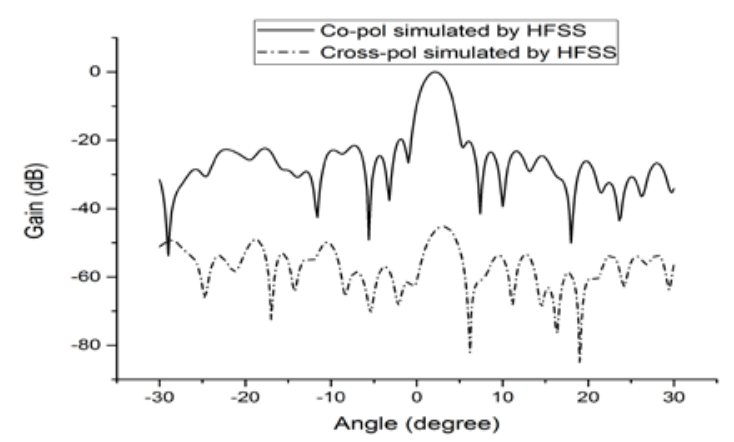

Fig. 5 Simulated radiation pattern in horizontal plane in $9.305 \mathrm{GHz}$

\section{Summary}

In this paper the calculation of cross-polarization in rectangular waveguide cross-polarization filter is reported. On this basis, 32-element narrow wall slotted waveguide array antenna in $9.305 \mathrm{GHz}$ with this filter is designed and simulated by HFSS. The level of cross-polarization lobes below the main lobe is lower than $-40 \mathrm{~dB}$ with the maximum side lobe level of $-20 \mathrm{~dB}$, allowing this antenna adapt to radar system.

\section{References}

[1]. M. Mazur, J. Wisniewski. "Performance of cross-polarization filter dedicated for slotted waveguide array antenna." IEEE National Radar Conference. Arlington, Virginia, USA, 10-14, May, 2010, p. 1335 - 1338.

[2]. L. B. Whitbourn, R. C. Compton. "Equivalent-circuit formulas for metal grid reflectors at a dielectric boundary. " Applied Optics. Vol. 24 (1985) No. 2, p. 217-220.

[3]. W. Wang, J. Jin, J. G. Lu, et al. "Waveguide slotted array antenna with broadband, dual-polarization and low cross-polarization for X-band SAR applications." IEEE International Radar Conference. Arlington, Virginia, USA, 9-12, May, 2005, p. 653-656.

[4]. A. Mallahzadeh, S. Mohammadalinezhad. "An Ultralow Cross-Polarization Slotted Waveguide Chebyshev Array Antenna." European Conference on Antennas and Propagation. Rome, Italy, 11-15, April, 2011, p. 1953-1956.

[5]. H. G. Booker, "Slot aerials and their relation to complementary wire aerials (Babinet's principle)." Electrical Engineers - Part IIIA: Radiolocation. Vol. 93 (1946) No. 4, p. 620-626. 
[6]. A. F. Stevenson, "Theory of Slots in Rectangular Wave - Guides." Journal of Applied Physics. Vol. 19 (1948) No. 1, p. 24-38. 